\title{
Application of shape memory alloy in Civil Engineering
}

\author{
Di-Cui ${ }^{1, a}$, Bo-Gan ${ }^{2, b}$ * \\ ${ }^{1}$ Research and development center of the civil engineering technology, Dalian University, Dalian, \\ China \\ ${ }^{2}$ College of Civil Engineering, Shenyang Jianzhu University, Shenyang, China; \\ acuidip@163.com, b276742213@qq.com
}

Keywords: Shape memory alloy ; civil engineering ; application

Abstract: Shape memory alloy is a new type of metal functional materials developed in recent decades. Because of its unique shape memory effect and super elasticity, etc., in many areas have a good application. In this paper, the basic properties of shape memory alloy and the application of shape memory alloy in the field of civil engineering are summarized. In the end, the existing problems and future development directions of the shape memory alloy in the field of civil engineering are pointed out.

\section{Introduction}

Shape memory alloy (Shape memory alloy, SMA) as one of the smart materials, due to large deformation can be recovered, in the restricted recovery can produce a great driving force, resistance is sensitive to strain, high damping properties, resistance fatigue performance, widely used in construction, aviation, aerospace, military and medicine etc. In the field of construction engineering, because the application characteristic of SMA material, SMA material is used in the seismic isolation structure design, structure vibration control, reinforcement and repair and structure detection. Made of SMA material made of various dampers, seismic isolation and shock absorption support, SMA concrete intelligent structure has achieved remarkable results.

\section{Performance of shape memory alloys}

\section{Shape memory effect}

Shape memory effect is one of the important characteristics of SMA material, the shape memory effect is that the solid material in the plastic deformation, after heating to a certain temperature, can be restored to the shape of the deformation. The shape memory effect of SMA material is realized by the martensitic transformation. One-way shape memory effect of SMA material is SMA material with a certain shape. At low temperature, the external force changes its shape, and then heated to room temperature, put it in a low temperature environment, its shape does not change. In addition to the one-way shape memory effect, Ni Ti alloy after certain processing, such as multiple heating-cooling cycle, constrained heating and aging can generate two-way shape memory effect and the whole memory effect. One-way, two-way memory effect and the whole process is shown in table 1.Now shape memory effect is more applied to smart concrete. 
Tab 1 A one-way, two-way and the whole memory effect

Low
Initial shape
shape
Twe-way
memory
whole memory

\section{Super-elastic properties}

In addition to the shape memory effect, SMA has a very important feature, that is, the super elasticity. Super elasticity means that when the ambient temperature at the end of the austenite phase transformation temperature above, the SMA material be applied external stress, until its production of plastic deformation, in the case of heating, the SMA material can be aslo restored to the original shape, that is, the original austenite state ${ }^{[1]}$. At present, many scholars are dedicated to the use of the super elasticity of SMA to carry out the structure of passive control.

\section{Damping characteristics}

The damping characteristic of SMA is due to the self coordination ability of the martensitic transformation and the hysteretic elastic migration of various interfaces (twin boundary, phase interface, variant Interface). During the martensitic transformation, due to the nucleation and growth of martensite, absorption of vibration energy is gradually increased, the size and vibration of the internal friction peak a week formed martensite is directly proportional to the number of . The damping of SMA is the smallest when the mother is in a state, and the full martensite state is second. When the mixed state of the parent phase and martensite phase, the damping is the largest. SMA can be used to combine the damping characteristics of other smart materials, to achieve the purpose of reducing vibration and noise ${ }^{[2]}$.

\section{Resistance properties}

The resistance of SMA can reflect the change of the internal material, which reflects the change of the content of each phase, and apperceive the temperature, stress and strain of the material. Because of the change of shape memory alloy, the resistance of SMA is very complex, and the variation of resistance of shape memory alloy is affected by the composition of material, stress, heat treatment and different application of different shapes. Experimental studies show that the resistance of the $\mathrm{Ni}-\mathrm{Ti}$ alloy has the following characteristics ${ }^{[3]}$ :(1) In the process of constant temperature loading and unloading, the resistance strain curve of Ni-Ti is slightly lower, but the range of the move is minuteness, and the resistance strain curve of each cycle is almost coincident;(2) After a certain cyclic loading and unloading test, the resistance strain curve of the Ni-Ti super elastic alloy wire will be stable. 


\section{Application status of shape memory alloy in civil engineering}

\section{Application and research of SMA in passive control of structures}

The application of SMA in the passive control of structure is mainly concentrated on the application of various dampers, and some research is to improve the quality of the structure by using the super elasticity of SMA, which makes the structure has higher resistance to the external force, and has a strong ability to self repair after the external force.

$\mathrm{Ni}$ Lifeng ${ }^{[4]}$ et al installed a good performance of SMA damper on the model of cable-stayed bridge. the results showed that the damper energy dissipation effect is obvious, the cable-stayed bridge with the same contrast not installed dampers, vibration response of the cable-stayed bridge model was significantly weakened.

He Silong ${ }^{[5]}$ et al heated a buried pre-strain of $1.8 \%$ of SMA wire reinforced concrete beams and study on the response of the beam under the static load and the impact load.The results show that the SMA wire can be applied to the structure of the structure, improve the structural strength and stiffness, reduce the structural static and dynamic response.

E.Effendy ${ }^{[6]}$ studied in combination with low shear SMA through the shear wall diagonally SMA device, and then applied load.The following conclusions are drawn: the residual deformation of the SMA shear wall is obviously larger than that of the SMA shear wall in the condition of the super elastic state.

Li hui ${ }^{[7]}$ et al produced two new type of SMA energy dissipation devices by using the super elastic properties of Ni-Ti alloy wire, which are tensile and shear type SMA. The experimental results show that the vibration damping effect of the shear type energy dissipation device is better than that of the tensile type damper.

\section{Application and research of SMA in active control of structures}

Kuang Yachuan, Ou Jinping ${ }^{[8]}$ developed a smart concrete beams with damage self repair function. The room temperature in the austenitic state of shape memory alloy and contains repairing adhesive repair fiber tube embedded in the concrete tension zone or easy to produce crack position, using shape memory alloy superelastic characteristics and limited recovery generation larger driving force characteristics, control and restore the structure and component of the deformation and deflection, reduce the impact of the typhoon, earthquake, structure to improve the anti disaster ability, ensure the safety; using adhesives for repairing of crack filling, repair characteristics, strength recovery of concrete, improve the durability of the structure. The experimental results show that the shape memory alloy significantly improves the deformation capacity of the beam. When the external force is gone, the deflection of the beam in the shape memory alloy is quick recovery, the crack is closed.

Cui $\mathrm{di}^{[9]}$ et al developed the SMA concrete beam is made into a concrete beam with the super elastic shape memory alloy wire as the main reinforcement in the concrete beam. The mechanical property of the test was carried out by using the single point load test, and the bearing capacity, the residual deformation and the residual crack of the beams were compared with the same size and the same ratio of the reinforced concrete beams. The influence of SMA on the mechanical properties of the beams was analyzed.The results show that the SMA strand can reduce the residual deformation and residual crack, improve the self repairing ability of concrete beams. Compared with ordinary reinforced concrete beams, the bearing capacity of SMA concrete beams increases more significantly.

Di Shengkui ${ }^{[10]}$ et al conducted a study on the SMA bridge by using the resistive conduction performance and the driving capability of the pre embedded SMA material in the bridge, achieving the purpose of monitoring and repairing cracks. In their experiments, the effect of slip between SMA wire and concrete is considered, and the results are pointed out that the pre tension should be controlled at $20 \% \sim 30 \%$ of the ultimate tensile strain. In the appropriate SMA pre stretching length, the appropriate increase of the cross-section area of the alloy wire can enhance the effect of the driver recovery. 
Many scholars have studied the application of SMA in the field of civil engineering, but the research is mainly focused on the structural passive control. And the research of SMA is still in the stage of theoretical derivation and experimental analysis.

\section{Discussion on the existing problems}

There are still many problems in the application of shape memory alloy to the structure:

(1) the use of large size SMA materials for research work. So far, most of the research work is based on the small diameter of SMA wire as the experimental material, because of the influence of SMA production and processing technology and price.

(2) the use of SME requires power supply, and the special nature of the temperature limits its scope of work;

(3) SMA is difficult to be machined, and the processing technology is not mature.At the same time SMA welding of dissimilar materials has also hindered the development of SMA.

\section{Acknowledgments}

This work was supported by the National Natural Science Foundation of China (Grant No. 51378085, 51008040 and 11302034), the Scientific Research Fund of Liaoning Provincial Education Department (Grant No. L2013465).

\section{References}

[1] Li Hongnan, Li Jun, Song Gangbing. The piezoelectric smart materials of civil engineering structure control [J]. Journal of building structures, 2005,26 (3): 1-8.

[2] Cui Di, Li Hongnan, song Gangbing.Ni-Ti shape memory alloy resistance properties of [J]. Journal of building materials, 2008,11 (5): 567 - 573

[3] Qiu Zixue. SMA sensing, driving performance and structural health monitoring technology research [J]. Nanjing University of Science and Technology, 2005, (5): 11 - 41

[4] Ni Lifeng, Li Qiusheng, Li Aiqun. Experimental study on the novel shape memory alloy damper [J]. earthquake engineering and engineering vibration, 2002,22 (3): 145-148.

[5] He Silong, Chen Mengshi,. Study on the self diagnosis and self adaptive intelligent structure system of reinforced concrete reinforced concrete with shape memory alloy [C]., China Materials Symposium. Beijing: Chemical Industry Press, 1996:876-881.

[6]E.Effendy, W.I.Liao, G.Song. Seismic Behavior of Low-Rise Shear Walls with SMA Bars[J]. Earth\&Space,2006,3:5-8.

[7] Lihui, Ou Jingping. Shape memory alloy passive dampers and seismic response control experimental study and analysis [J]. Journal of building structures, 2005,26 (3): 35-44.

[8] Ou Jinping, Kuang adtran. Shape memory alloy reinforced concrete elastic beam deformation characteristics [J]. Building science and Engineering Journal, 2007,24 (3): 1 - 6.

[9] Cui Di, Li Hongnan, Song Gangbing. Shape memory alloy concrete beam mechanical performance test [J]. Engineering mechanics, 2010,27 (2): 117-123.

[10] Di Shengkui, Pan huawei, Sheng Jiwei. State constraints of SMA concrete beam crack monitoring and self repairing [J]. Journal of building materials, 2010,2:237-242. 